\section{Do dual-cure bulk-fill resin composites reduce gaps and improve depth of cure?}

May Anny Alves Fraga (D) 1, Lourenço Correr-Sobrinho (D) 1, Mário Alexandre Coelho Sinhoreti (D) 1, Talita Malini Carletti (D) 1, Américo Bortolazzo Correr'1D.

This in vitro study aimed to evaluate the gaps length and depth of cure of dualcure bulk-fill resin composites inserted in box-shaped preparations. Boxshaped preparations ( $4 \mathrm{~mm}$ deep) were made in fifteen human third-molars and divided into three groups according to the resin composites $(n=5)$ : Dualcure bulk-fill BulkEZ (BEZ); Dual-cure bulk-fill HyperFIL (HF); and Tetric Evoceram Bulk-fill (TETRIC), as control. Gaps length (\%) was evaluated in toothrestoration interface with micro-computed tomography $(\mu \mathrm{CT})$. The restorations were sectioned, and the degree of conversion (DC) and Knoop microhardness were evaluated at five depths $(0.3,1,2,3$, and $4 \mathrm{~mm})$. Microhardness data were statistically evaluated using absolute values (KHN) and relative values (microhardness percentages in relation to top). Gaps length (\%) increased in the following order: $\mathrm{BEZ}=\mathrm{TETRIC}<\mathrm{HF}$. The microhardness percentages in relation to top significantly decreased from $2 \mathrm{~mm}$ for TETRIC and $3 \mathrm{~mm}$ for HF. BEZ had constant microhardness and DC at all depths, while HF and TETRIC presented a significant decrease on DC at $4 \mathrm{~mm}$. Dual-cure bulk-fill composites did not reduce gaps compared to light-cure bulk-fill, but they can improve depth of cure of bulk-filled restorations.

\author{
${ }^{1}$ University of Campinas - Piracicaba Dental \\ School, Department of Restorative Dentistry, \\ Dental Materials Division.
}

Correspondence: May Anny Alves Fraga, DDS, MSc Department of Restorative Dentistry Dental Materials Division 901, Limeira Avenue, Piracicaba, SP, BR. ZIP Code 13414-903 Tel.: +55 1921065347

E-mail:mayannyfraga@hotmail.com

\title{
Introduction
}

Bulk-fill resin composites are direct restorative materials designed to be used in 4-5 $\mathrm{mm}$ increments (1). The increased depth of cure of bulk-fill composites occurs due to the high translucency given by the enlargement size of fillers particles and to the similar refractive index between organic matrix and fillers; additionally, the inclusion of new photoinitiator systems may contribute to improve the polymerization depth of the resin composites (2). Nevertheless, the amount of light transmitted to the bottom of the bulk-fill resin composites for some materials seems to be insufficient to guarantee a suitable degree of conversion in deep regions (3). Also, the absorbance of alternative photoinitiators is close to the violet range (380-420 nm), and it is characterized by light transmission only in the superficial layers of the resin composites (4).

Some changes were made to improve the light transmission of bulk-fill composites. Prepolymerized polymers were added to match the refractive index of fillers and organic matrix. Alternative monomers were added to reduce polymerization stress at the tooth-restoration interface. Such changes included higher molecular weight monomers, flexible monomers, and monomers capable of undergoing chain transfer fragmentation (5). However, bulk-fill composites still face problems generated by shrinkage stress, and gaps are present mainly in the bottom region of restoration (6).

Recently, dual-cure bulk-fill resin composites have been introduced as paste-paste fluid materials (7). The aim is to minimize the effects of light attenuation by chemical activation (3). Photoactivation enables fast and early curing of composites at superficial layers, while in the deepest layers, where light is attenuated, polymerization supplementation occurs by slower chemical cure (8). Moreover, the lower rate of polymerization before light curing allows a viscoelastic flow and stress relaxation within the material, which has been shown to reduce the gap in dual-cure resin composites (7). In addition, the commercial paste-paste fluid presentation could present a lower elastic modulus, which may reduce the polymerization shrinkage stress (9). Although promising outcomes have been found in dual curing resin composites (7), studies that evaluated these materials in human teeth with box-shaped preparations were not found. In Class I posterior teeth restorations, the effect of polymerization shrinkage stress is 
even more exacerbated since the preparations require larger volumes of composite and present a high C-factor (10).

Therefore, this study aimed to evaluate the gaps and depth of cure of dual-cure bulk-fill composites placed in box-shaped preparations. The tested hypotheses of this study were: $\{1\}$ Dual-cure bulk-fill composites would reduce the gaps length (\%) when compared to the photoactivated bulk-fill resin composites; $\{2\}$ Dual-cure bulk-fill resin composites would have a higher depth of polymerization (degree of conversion and microhardness) than photoactivated bulk-fill resin composites.

\section{Materials and methods}

\section{Box-shaped preparation}

The local Research Ethics Committee (protocol No. 94624818.6.0000.5418) approved this study. A pilot study was performed considering gap evaluation percentage using three samples per group, observing a difference in means $=15 \%$ and standard deviation $=8 \%$. The 0.80 power and $5 \%$ level of significance were considered. The minimum sample size for each group was five. Software $G$ Power 3.1.9.2 (Franz Faul, Universität Kiel, Germany) was used to calculate data.

Fifteen extracted human third molars were cleaned and stored in a refrigerator at $4{ }^{\circ} \mathrm{C}$ up to 3 months after extraction. The tooth roots were sectioned $2 \mathrm{~mm}$ below the cementoenamel junction using a diamond disc mounted in a cutting machine (Isomet, Buehler Ltd., Lake Buff, IL, USA).

Box-shaped preparations were made in the teeth using the protocol proposed by Silame et al. (11) (Figure 1). Briefly, the teeth were positioned upside down to allow the box-shape preparation. The directions of preparations followed from pulp chamber to enamel. To guarantee that the floor of all preparations was located in the deep dentin, the distance between enamel and pulp chamber roof was obtained with a manual thickness gage (Minellium, São Caetano do Sul, SP, BR). The following equation was applied to each tooth to set up the depth of wear of dentin:

$$
\mathrm{M}_{\text {Dentin }}=(\mathrm{E}-2 \mathrm{~mm}) / 3
$$
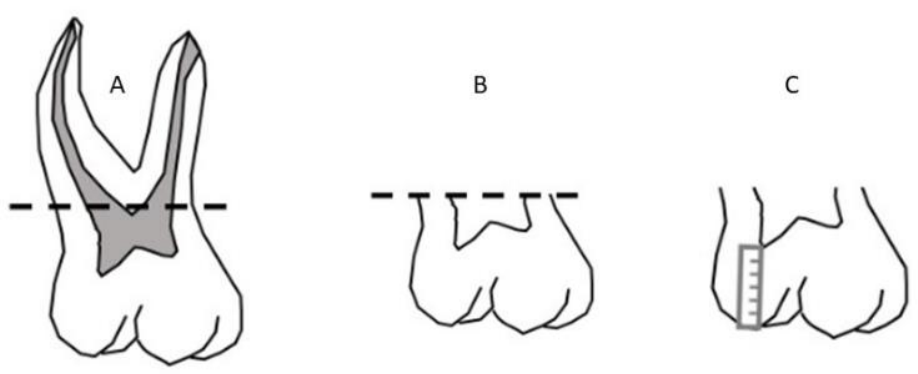

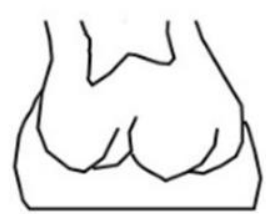

Figure 1. Schematic representation of the box-shaped preparations. A. Dashed line represent the site where crown-to-root was separated $2 \mathrm{~mm}$ below the cementum-enamel junction. B. Crown after removal of the roots $C$. the thickness between the pulp chamber roof and the occlusal surface was measured. D. Resin composite of placed on the occlusal face of the crown, and resin composite was planned. E. Box-shaped preparations were made in the pulp chamber. F. Final aspects of preparations after polishing with $\mathrm{F}$ and $\mathrm{FF}$ tips. 
Where $M_{\text {Dentin }}$ corresponds to the deep dentin; " $E$ " is the total distance between enamel and pulp chamber. Two millimeters $(2 \mathrm{~mm})$ was considered as the mean enamel thickness of the teeth.

The occlusal surfaces of the teeth were restored with Scothbond Universal (3M/ESPE, St. Paul, MN, USA) and Filtek Z350 resin composite (3M/ESPE, USA). A leveling press (Leitz, Wetzlar, Germany) was used to plan the resin composites' surface and make its occlusal surface parallel to the axial wall of preparation. The specimens were fixed to the base of the machine (Elquip, São Carlos, SP, Brazil), and box-shaped preparations ( $4 \mathrm{~mm}$ width $\times 6 \mathrm{~mm}$ long and $4 \mathrm{~mm}$ deep) were made with cylindrical diamond burs \# 3099 (KG Sorensen, Sao Paulo, SP, Brazil). The burs were discarded after five prepared teeth. The preparations were refined with $\mathrm{F}$ and $\mathrm{FF}$ diamond tips of the same numbering and washed with water. The C-factor was approximately 4.30, and the preparation volume was $96 \mathrm{~mm}^{3}$.

\section{Restorative procedure}

The Clearfil SE Bond 2 (Kuraray Noritake Dental, Tokyo, Japan) adhesive system was applied according to manufacturer's instructions in all preparations. The primer was actively applied for $20 \mathrm{~s}$ and gently air-dried for $20 \mathrm{~s}$. The bond was applied, gently air-dried, and photoactivated for $10 \mathrm{~s}$ with a multiple-peak LED device (Bluephase G2, Ivoclar Vivadent, Schaan, Liechtenstein, $960.0 \pm 7.0 \mathrm{~mW} / \mathrm{cm}^{2}$ radiant exitance). Three commercial resin composites were investigated $(n=5)$ : two dual-cure bulk-fill resin composites, BulkEZ (BEZ) (Danville Materials, Carlsbad, CA, USA) and HyperFIL (HF) (Parkell, New York, NY, USA); and a photoactivated bulk-fill resin composite (TETRIC) (Tetric N-ceram Bulk-fill, Ivoclar Vivadent, Liechtenstein), as control. A detailed description of the materials is presented in Table 1.

Table 1. Description of the composite resins, abbreviations used in the groups, composition and photoactivation procedures.

$\begin{array}{llll}\text { Material/Shade (Code/Lot No). } & \begin{array}{l}\text { Abbreviation used in } \\ \text { text / Classification }\end{array} & \text { Composition (filler wt } \% \text { \& vol\%) } & \begin{array}{l}\text { Photoactivation } \\ \text { protocol }\end{array}\end{array}$

\begin{tabular}{|c|c|c|c|}
\hline \multirow{2}{*}{$\begin{array}{l}\text { Bulk EZ/A2 (LOK75. 94776) } \\
\text { Danville Materials, Carlsbad, CA, } \\
\text { USA }\end{array}$} & $\begin{array}{l}\text { BEZ/ Dual-cure bulk- } \\
\text { fill }\end{array}$ & $\begin{array}{l}\text { Monomers: EBPDMA, TEGDMA, } \\
\text { BisGMA, UDMA }\end{array}$ & \multirow{2}{*}{$\begin{array}{c}10 \mathrm{~s} / 960 \mathrm{~mW} / \mathrm{cm}^{2}-90 \\
\text { seconds after paste } \\
\text { mixing }\end{array}$} \\
\hline & composite & $\begin{array}{l}\text { Fillers: Barium glass, Ytterbium } \\
\text { Trifluoride (50-70 wt } \%)\end{array}$ & \\
\hline \multirow{2}{*}{$\begin{array}{l}\text { HyperFIL TM DC/ Universal } \\
\text { (1823518200) Parkell, New York, } \\
\text { NY, USA }\end{array}$} & $\begin{array}{l}\text { HF/ Dual-cure bulk- } \\
\text { fill }\end{array}$ & $\begin{array}{l}\text { Monomers: BisEMA, UDMA, and } \\
\text { other dimethacrylate monomers. }\end{array}$ & \multirow{2}{*}{$40 \mathrm{~s} / 960 \mathrm{~mW} / \mathrm{cm}^{2}$} \\
\hline & composite & $\begin{array}{l}\text { Fillers: Barium glass/silica (70-75 } \\
\text { wt\%) }\end{array}$ & \\
\hline \multirow{2}{*}{$\begin{array}{l}\text { Tetric N-ceram Bulk-fill/IVA (X- } \\
\text { 27163) Ivoclar Vivadent AG, } \\
\text { Schaan, Liechtenstein }\end{array}$} & Tetric / Light-cure & $\begin{array}{l}\text { Monomers: BisGMA, BisEMA, } \\
\text { UDMA }\end{array}$ & \multirow[b]{2}{*}{$20 \mathrm{~s} / 960 \mathrm{~mW} / \mathrm{cm}^{2}$} \\
\hline & composite & $\begin{array}{l}\text { Fillers: Barium aluminium silicate } \\
\text { glass, Ytterbium Trifluoride, } \\
\text { Prepolymer, Mixed Oxides ( } 77 \\
\text { wt } \%, 55 \text { vol } \% \text { ) }\end{array}$ & \\
\hline \multirow{2}{*}{$\begin{array}{l}\text { Filtek Z350/A2E (1826900729) } \\
\text { Irvine, CA, EUA }\end{array}$} & $\begin{array}{l}\text { Z350/ Light-cure } \\
\text { incremental }\end{array}$ & $\begin{array}{l}\text { Monomers: BisGMA, UDMA, } \\
\text { TEGDMA, PEGDMA, BisEMA. }\end{array}$ & \multirow{2}{*}{$20 \mathrm{~s} / 960 \mathrm{~mW} / \mathrm{cm}^{2}$} \\
\hline & composite & $\begin{array}{l}\text { Fillers: Zirconia/silica (72.5 wt } \% \text {, } \\
63.3 \text { vol\%) }\end{array}$ & \\
\hline
\end{tabular}

Abbreviations: BisGMA, bisphenylglycidyl dimethacrylate; BisEMA, ethoxylated bisphenol-A dimethacrylate; EBPDMA , 1,6-bis[2-methacryloyloxyethoxycarbonylamino]-2,4,4- trimethlhexane; PEGDMA, Polyethylene glycol dimethacrylate; UDMA, urethane dimethacrylate; TEGDMA, triethylene glycol dimethacrylate.

The restorative procedures were made according to directions of manufacturers. BEZ was mixed with an automix tip. The first portion of the mixed resin composite was discarded to avoid bubbles in the restoration. BEZ was inserted in bulk into the Class I box-shaped preparations. After 90 seconds delay, 
BEZ was photoactivated for 10 seconds (Bluephase G2, Ivoclar Vivadent, Liechtenstein - $960.0 \pm 7.0$ $\mathrm{mW} / \mathrm{cm}^{2}$ radiant exitance). HF composite was manually mixed for 10 seconds in a glass plate and inserted into the preparation with a spatula. Photoactivation was performed for 40 seconds (Bluephase G2, Ivoclar Vivadent, Liechtenstein) immediately after inserting the composite into the preparation. TETRIC was inserted in bulk and photoactivated for 20 seconds (Bluephase G2, Ivoclar Vivadent, Liechtenstein). Photoactivation was performed on the top of restorations in all groups.

After the restorative procedures, all specimens were stored in deionized water for $24 \mathrm{~h}$ at $37^{\circ} \mathrm{C}$. Internal gap, microhardness, and degree of conversion were evaluated sequentially in the same specimen, as described below.

\section{Gap evaluation}

Internal gaps were evaluated with micro-computed tomography $(\mu \mathrm{CT})$ (SkyScan 1272, Bruker, Belgium) by a calibrated examiner. Images were acquired using $100 \mathrm{kV}, 100 \mathrm{uA}$, and a $0.11 \mathrm{~mm}$ cooper filter with a pixel size of $10 \mu \mathrm{m}$. The specimens were scanned for 180 degrees with 2 theta angles and step of 1.0 degree.

Data were exported to DICOM format, and the internal gaps were quantified by ITK-SNAP software (12). The percentage of gaps was evaluated in 2D images every $400 \mu \mathrm{m}(13)$ in vestibule-lingual direction. The marginal gap percentage was calculated using the formula: Gaps $(\%)=100 \times$ (gap extension/preparation perimeter). The gaps were considered as areas of discontinuity of bonding at the tooth-restoration interface. A single calibrated examiner (intra-examiner correlation index $=96 \%$ ) measured all images. The gaps were considered as areas of discontinuity of bonding at the toothrestoration interface. Ten different regions were randomly selected and measured twice (baseline and after ten days), and the intra-examiner correlation (ICC) was calculated by SPSS software (IBM SPSS Statistics 21) at $\alpha=0.05$.

\section{Microhardness}

After $\mu \mathrm{CT}$ analysis, the specimens were cut axially in their centers, in an occlusal-cervical direction, using a water-cooled diamond disc (Isomet Diamond Wafering Blades, No. 11-4244, Buehler Ltd., Lake Buff, IL, USA). Both halves were wet ground and polished using SiC abrasive papers (1000-, 1200- and 2000-grit, Norton Abrasives, Vinhedo, SP, Brazil). Knoop hardness was evaluated in the microdurometer HMV 2000 (Shimadzu, Tokyo, Japan), with a static load of 50gF (0.490 N) and 10 seconds indentation time. Three indentations were made in each depth of restoration $(0.3 \mathrm{~mm}, 1 \mathrm{~mm}, 2 \mathrm{~mm}, 3 \mathrm{~mm}$, and 4 $\mathrm{mm})$, and a mean value calculated for each depth. The indentations were initiated at $0.3 \mathrm{~mm}$ from the top of the restoration to avoid the resin composite's oxygen-inhibited surface layer. The microhardness percentage $(\mathrm{MH} \%)$ values at $1 \mathrm{~mm}, 2 \mathrm{~mm}, 3 \mathrm{~mm}$, and $4 \mathrm{~mm}$ were calculated in relation to the top according to the following equation: $\mathrm{MH} \%=(\mathrm{MH} \text { depth/MH top })^{*} 100$.

\section{Degree of conversion}

Degree of conversion was evaluated at the same depths used for microhardness test. The analysis was performed in a micro-Raman (XploRA, Horiba, Kyoto, Japan), using a $785 \mathrm{~nm}$ laser. The initial reference spectrum was collected from the uncured composite. For the dual-cure composite groups, the components were mixed on a glass slide for 5 seconds, and the spectra were collected for 20 seconds after mixing. The software (SpectraGryph 1.2) measured the band areas of phenyl CC peak at $1610 \mathrm{~cm}-1$ and the vinyl CC peak at $1640 \mathrm{~cm}-1$. The equation calculated the degree of conversion:

Degree of conversion $=100 \times\left[1-\left(R \_\right.\right.$polimerization $) /($R_unpolymerized $\left.)\right]$

where " $R$ " is the peak absorption area ratio at $1640 \mathrm{~cm}^{-1} / 1610 \mathrm{~cm}^{-1}$.

\section{Statistical Analyses}

The statistical analyses were carried out using SPSS software (IBM SPSS Statistics 21), considering a significance level of 5\%. Data were analyzed for normality with the Shapiro-Wilk test. Levene's test was used to analyze the homoscedasticity of variances. The gap formation was analyzed by one-way ANOVA and Tukey's post-hoc test. The split-plot ANOVA and Bonferroni's post-hoc tests were used to compare degrees of conversion, $\mathrm{KHN}$, and $\mathrm{MH} \%$. 


\section{Results}

The percentage of internal gaps was significantly different among the resin composites $(p=0.010)$ (Table 2). HF presented higher mean values of percentage of gaps $(52.54 \% \pm 10.60)$ than other resin composites. There was no significant difference between TETRIC $(38.3 \% \pm 4.1)$ and BEZ $(31.8 \% \pm 7.6)$.

Table 2 - Mean value and standard deviation of gaps (\% length of entire internal interface).

\begin{tabular}{cc}
\hline Groups & Gap (\% total interface length) \\
\hline BEZ & $31.9(7.6) \mathrm{b}$ \\
HF & $52.5(10.6) \mathrm{a}$ \\
TETRIC & $38.4(4.1) \mathrm{b}$
\end{tabular}

${ }^{*}$ Different letters indicate statistically differences among resin composites $(p \leq 0.05)$.

The outcomes of microhardness percentage in relation to the top can be seen in Figure 2. ANOVA split-plot test for microhardness percentage showed that interaction between factors (materials*depthof-cure) was significant ( $p=0.017)$. Microhardness percentage of BEZ was constant from the top to the bottom of the restorations. TETRIC and HF had a significant decrease in microhardness percentages from $2 \mathrm{~mm}$ and $3 \mathrm{~mm}$, respectively. In $4 \mathrm{~mm}$ of depth, BEZ was significantly higher than HF and TETRIC. The absolute mean values of microhardness can be seen in Table 3. BEZ presented similar hardness in all depths. The microhardness of HF in $4 \mathrm{~mm}$ was significantly lower than in $0.3 \mathrm{~mm}, 1 \mathrm{~mm}$, and $2 \mathrm{~mm}$. For TETRIC, KHN in $2 \mathrm{~mm}$ and $3 \mathrm{~mm}$ was significantly lower than in $0.3 \mathrm{~mm}$ and $1 \mathrm{~mm}$; the KHN in $4 \mathrm{~mm}$ was significantly lower than in other depths.

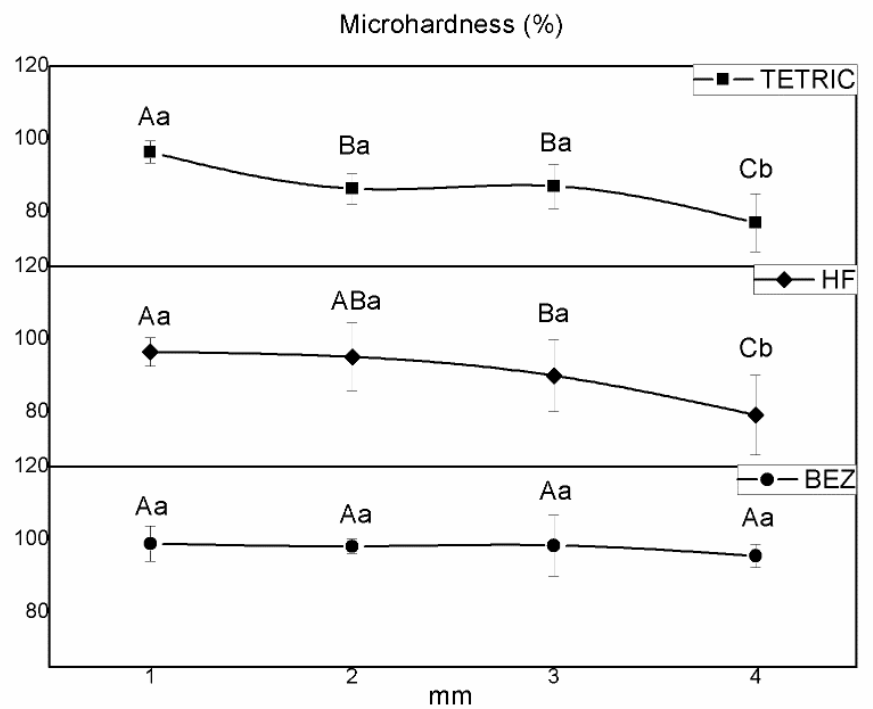

Figure 2. Microhardness percentages (mean and standard deviation) in relation to top in different depths of the restoration. Different capital letters represent significant differences between depths within the same resin composite ( $\mathrm{p} \leq 0.05)$. Different lowercase letters represent significant differences between resin composites within the same depth ( $p$ $\leq 0.05)$.

Table 3. Microhardness (KHN) mean values (standard deviation) of resin composites in different depths.

\begin{tabular}{cccccc}
\hline & $0.3 \mathrm{~mm}$ & $1 \mathrm{~mm}$ & $2 \mathrm{~mm}$ & $3 \mathbf{m m}$ & $4 \mathrm{~mm}$ \\
\cline { 2 - 6 } BEZ & $46.2(8.4) \mathrm{A}$ & $45.1(8.1) \mathrm{A}$ & $44.4(10.5) \mathrm{A}$ & $44.7(6.2) \mathrm{A}$ & $45.4(10.7) \mathrm{A}$ \\
HF & $59.9(8.1) \mathrm{A}$ & $57.7(10.5) \mathrm{A}$ & $56.8(6.2) \mathrm{A}$ & $53.3(10.7) \mathrm{AB}$ & $46.9(12.7) \mathrm{B}$ \\
TETRIC & $65.4(10.5) \mathrm{A}$ & $62.6(6.2) \mathrm{A}$ & $58.8(10.7) \mathrm{B}$ & $58.2(12.7) \mathrm{B}$ & $52(14.7) \mathrm{C}$
\end{tabular}

* Different capital letters represent significant differences among depths for each resin composite ( $p \leq 0.05)$. 
The depth did not influence the degree of conversion of BEZ. However, HF and TETRIC had a significant decrease in degree of conversion at $4 \mathrm{~mm}$ depth (Figure 3). TETRIC presents the lower degree of conversion mean values among the groups in all depths. HF showed a higher degree of conversion mean values than BEZ in $0.3 \mathrm{~mm}, 1 \mathrm{~mm}, 2 \mathrm{~mm}$, and $3 \mathrm{~mm}$, although in $4 \mathrm{~mm}$, the mean values were similar between $\mathrm{HF}$ and BEZ.

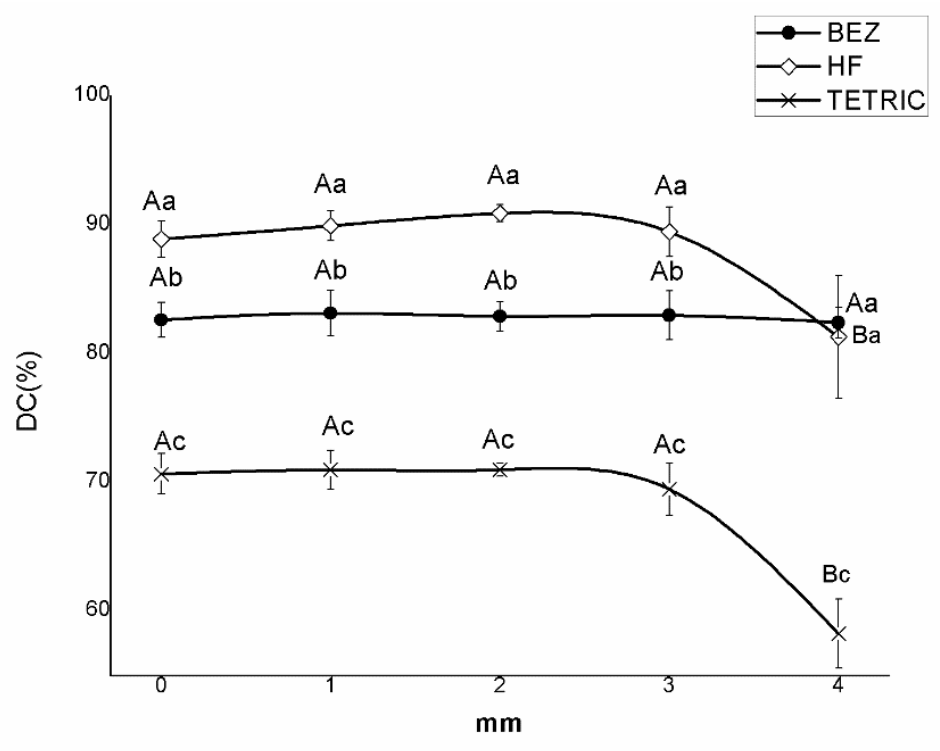

Figure 3. Degree of conversion (DC) percentages (mean and standard deviation). Different capital letters represent significant differences between depths within the same material $(p \leq 0.05)$.

\section{Discussion}

The first hypothesis that all dual-cure bulk-fill resin composites would reduce the gaps at toothrestoration interface was rejected since the dual-cure bulk-fill composites presented similar or higher percentages of gaps than the light-cure composite TETRIC. The depth of cure (microhardness and degree of conversion) at the bottom of the HF restorations was lower than the top. Thus, the second hypothesis that the depth of cure would be improved using dual-cure bulk-fill composites was rejected.

Dual-cure bulk-fill composites have emerged on the market as an alternative to minimize internal gaps and improve depth of polymerization. The chemical cure is a slower process, reduces polymerization shrinkage stress, and minimizes the polymerization deficiency at the bottom of the restoration by light attenuation $(3,14)$. Following manufacturer's recommendation, the dual-cure BEZ composite was photoactivated only 90 seconds after mixing. The delay in photoactivation of dual-cure composites could be responsible for reducing gap formation once it may reduce polymerization shrinkage stress independently of the degree of conversion $(14,15)$. When photoactivation is delayed, material autoacceleration occurs more slowly by the action of free radicals formed by the oxy-reduction reaction, postponing the vitrification of the material (16). Since volumetric shrinkage, elastic modulus, and Cfactor are also relevant factors for decreasing polymerization stress (17), the gel point delay alone may not be capable of inhibiting gap formation. Despite this effect, BEZ did not reduce gaps than TETRIC; nevertheless, this result may be minimized as TETRIC is a photoactivated bulk-fill composite, showing the best performance on gap formation $(6,7)$. In addition, both materials may have formed gaps due to the insertion technique itself, where bulky increments are used, increasing the adaptation difficulty and the polymerization shrinkage stress (18). These gaps occur majorly in base and preparation angle due to the shrinkage stress vectors and difficulty adapting material. The last is related to the insertion technique and viscosity of the material (7). A previous study reported lower gaps in BEZ compared to light-cure bulk-fill composites (7). Some issues may be discussed about differences in our findings. In the previous study (7), the resin composite was bonded on a resin composite cylinder in which the surface was sandblasted with alumina particles and silanated before adhesive system application. This procedure could have favored the adhesion and consequently reduced the effects of composite polymerization shrinkage stress. In addition, the preparation walls were thin ( $0.5 \mathrm{~mm}$ floor vs. $2 \mathrm{~mm}$ axial walls), allowing 
for higher compliance. In our study, a box-shaped preparation was used. The adhesion was made on dentin, a more complex substrate due to moisture and high organic content, which jeopardizes bonding effectiveness and favors the environment for gap formation. In addition, we evaluated the gaps 24 hours after storage in water, while in the previous study (7), the gaps were evaluated in real-time, not considering the late double bond conversion and polymerization shrinkage.

When dual-cure bulk-fill composites were compared, BEZ presented lower internal gap percentages than HF. Although HF is a dual-cure the manufacturer has suggested composite, no delay in photoactivation. Since the light-activation was performed immediately after the insertion of material into the preparation, the polymerization kinetics becomes similar to a photoactivated bulk-fill composite (17). Additionally, free radicals continue to be formed by the oxy-reduction reaction up to 24 hours after photoactivation (19). After the gel point, the volumetric shrinkage caused by the subsequent conversion of carbon double bonds will not enable stress dissipation since matrix is already in a vitrification state (20), which might lead to stress concentration at the bonding interface.

The degree of conversion also influences the polymerization shrinkage stress. Higher conversion degree values benefit the mechanical properties; however, the higher monomeric conversion results in high volumetric shrinkage and polymerization shrinkage of stress (21). The dual-cure bulk-fill composites presented a higher degree of conversion. This may be due to the presence of two distinct polymerization reaction initiation processes: 1 . An oxy-reduction chemical polymerization, when the initiator molecule contacts an accelerator, and 2. a physical polymerization activated by light when free radicals are formed from a dissociated molecule stimulated by a specific wavelength $(3,15)$. The light may be dispersed, absorbed, or transmitted through the resin composite. In bulk-fill light cure resin composites, the amount of light transmitted to the bottom of restoration may be insufficient to guarantee a suitable degree of conversion. On the contrary, the chemical activation of dual-cure resin composites might enable a suitable degree of conversion in deep areas of the restoration even when a lower light radiant exposure was used.

When dual curing bulk-fill resin composites were compared, HF showed more gaps and also a higher degree of conversion up to $3 \mathrm{~mm}$ depth than BEZ. HF was photoactivated for 40 seconds, resulting in a total radiant exposure of $38.4 \mathrm{~J} / \mathrm{cm}^{2}$. It may have contributed to a higher degree of conversion on top of the restoration and, consequently, more gap formation. Although BEZ has a lower degree of conversion than HF, the cure profile was homogeny, and fewer gaps were observed. According to the manufacturer, BEZ self-cure presents a patented initiator, enabling a reduced polymerization shrinkage (22). Additionally, when compared to TETRIC, BEZ showed a more significant degree of conversion, and the percentage of gaps was similar to TETRIC, evidencing the advantage of BEZ to dissipate stress.

Although HF showed a high degree of conversion, polymerization was not homogeneous. Also, microhardness and degree of conversion decreased with depth. The same behavior was observed in TETRIC that also reduced microhardness and degree of conversion from $2 \mathrm{~mm}$ depth. It corroborates with a previous study (8) that demonstrated similar kinetic patterns for both materials. The bottom-to-top ratio frequently estimates depth of cure of resin composite materials, and, at a ratio inferior to $80 \%$, they are not adequately cured (4). On the other hand, our results corroborate with previous studies showing polymerization homogeneity of BEZ in all depths of restoration $(8,23)$. We can hypothesize that HF is more dependent on light while BEZ depends on chemical activation. Again, the high radiant exposure $\left(38.4 \mathrm{~J} / \mathrm{cm}^{2}\right)$ and absence of delay after mixing to photoactivation of $\mathrm{HF}$ may maximize degree of conversion during photoactivation and the chemical activation was not able to improve polymerization in deep layers. On the contrary, the maximum rate of polymerization and vitrification of BEZ may have occurred during the delay time (chemical activation).

Concurrently, the monomeric composition and percentage of filler particles may have contributed to the lower microhardness of BEZ. Microhardness is a mechanical surface property closely associated with wear resistance (24). The low mechanical performance might restrict the use of BEZ at the top of the restoration, requiring the use of a composite resin cap with regular consistency, as previously reported for other bulk-fill flowable composites (25).

Dual-cure bulk-fill composites are recent in the market, and further studies should investigate the real effectiveness of these materials in vivo. In the present study, no decrease in gap formation was seen in dual bulk-fill composite. However, BEZ group has shown improvements in polymerization homogeneity. The application of BEZ, as a base material of restorations, in less bulky increments has not been explored and could be a promising alternative in reducing gap and polymerization homogeneity. In addition, BEZ demonstrated lower cusp flexion after a 2 min delayed without reducing the degree of conversion (14). Further studies are needed to validate the results of this study, specially using delayed 
photoactivation with different dual-cure bulk-fill. Therefore, it can be concluded that gap formation is material-dependent. Dual-cure bulk-fill composites did not reduce gap compared to light-cure bulk-fill, but depth of cure was improved with dual-cure resin composites.

Acknowledgment

The authors thank the Laboratory of Soft Materials staff at the Brazilian Laboratory of Nanotechnology (LNNano - CNPEM) for X-ray Micro-CT analysis and instrumentation. This study was supported by Conselho Nacional de Desenvolvimento Científico e Tecnológico - CNPq (Grant 309237/2019-0) and Coordination for the Improvement of Higher Education Personnel (CAPES) [Code 001].

\section{Resumo}

0 objetivo neste estudo in vitro foi mensurar as fendas e a profundidade de polimerização de compósitos resinosos bulk-fill duais inseridos em preparos em forma de caixa. Os preparos em forma de caixa ( $4 \mathrm{~mm}$ de profundidade) foram realizados em quinze terceiros molares e separados em 3 grupos, de acordo com o compósito resinoso $(n=5)$ : Bulk-fill dual BulkEZ (BEZ); Bulk-fill dual HyperFIL (HF); Tetric Evoceram Bulk-fill (TETRIC). 0 comprimento das fendas (\%) foi avaliado na interface denterestauração por meio de micro-tomografia computadorizada $(\mu \mathrm{CT})$. As restaurações foram seccionadas e o grau de conversão (GC) e microdureza Knoop foram avaliados em cinco profundidades $(0,3,1,2,3$ e 4 $\mathrm{mm})$. Os dados de microdureza foram avaliados estatisticamente por meio de valores absolutos e relativos (porcentagens de microdureza em relação ao topo). 0 comprimento das fendas (\%) aumentou na seguinte ordem: BEZ = TETRIC $<$ HF. 0 percentual de microdureza em relação ao topo diminuiu significativamente a partir de $2 \mathrm{~mm}$ para TETRIC e $3 \mathrm{~mm}$ para HF. BEZ apresentou percentuais de microdureza e de GC constantes em todas as profundidades, enquanto HF e TETRIC apresentaram decréscimo significativo no $\mathrm{GC}$ em $4 \mathrm{~mm}$. Os compósitos resinosos bulk-fill duais não reduziram as fendas formadas quando comparados a um compósito bulk-fill fotopolimerizável; entretanto, os mesmos podem melhorar a profundidade de polimerização em restaurações de incremento único. 


\section{References}

1. Kim RJ, Kim YJ, Choi NS, Lee IB. Polymerization shrinkage, modulus, and shrinkage stress related to tooth-restoration interfacial debonding in bulk-fill composites. J Dent 2015;4:430-439.

2. Son SA, Park JK, Seo DG, Ko CC, Kwon YH. How light attenuation and filler content affect the microhardness and polymerization shrinkage and translucency of bulk-fill composites? Clin Oral Investig 2017;21:559-565.

3. Vandewalker JP, Casey JA, Lincoln TA, Vandewalle KS. Properties of dual-cure, bulk-fill composite resin restorative materials. Gen Dent 2016;64:68-73.

4. Rocha MG, de Oliveira D, Sinhoreti M, Roulet JF, Correr AB. The Combination of CQ-amine and TPO Increases the Polymerization Shrinkage Stress and Does Not Improve the Depth of Cure of Bulk-fill Composites. Oper Dent 2019;44:499-509.

5. Fugolin APP, Pfeifer CS. New Resins for Dental Composites. J Dent Res 2017;96:1085-1091.

6. Fronza BM, Rueggeberg FA, Braga RR, Mogilevych B, Soares LE, Martin AA, Ambrosano G, Giannini M. Monomer conversion, microhardness, internal marginal adaptation, and shrinkage stress of bulk-fill resin composites. Dent Mater 2015;31:1542-1551.

7. Hayashi J, Espigares J, Takagaki T, Shimada Y, Tagami J, Numata T, Chan D, Sadr A. Real-time indepth imaging of gap formation in bulk-fill resin composites. Dent Mater 2019;35:585-596.

8. Wang R, Liu H, Wang Y. Different depth-related polymerization kinetics of dual-cure, bulk-fill composites. Dent Mater 2019;35:1095-1103.

9. Ferracane JL. Resin-based composite performance: are there some things we can't predict? Dent Mater 2013;29:51-58.

10. Stavridakis MM, Kakaboura Al, Ardu S, Krejci I. Marginal and internal adaptation of bulk-filled Class I and Cuspal coverage direct resin composite restorations. Oper Dent 2007;32:515-523.

11. Silame FDJ, Geraldeli GP, Sinhoreti MAC, Pires-de-Souza FCP, Roulet JF, Geraldeli S. Dentin $\mu$ TBS and Hardness of Bulk-fill and Conventional Composites Placed in a Box-shaped Cavity Preparation J Adhes Dent 2017;19:395-400.

12. Yushkevich PA, Piven J, Hazlett HC, Smith RG, Ho S, Gee JC, Gerig G. User-guided 3D active contour segmentation of anatomical structures: significantly improved efficiency and reliability. Neuroimage 2006;3:1116-1128.

13. Han SH, Sadr A, Tagami J, Park SH. Internal adaptation of resin composites at two configurations: Influence of polymerization shrinkage and stress. Dent Mater 2016;32:1085-1094.

14. Hughes KO, Powell KJ, Hill AE, Tantbirojn D, Versluis A. Delayed Photoactivation of Dual-cure Composites: Effect on Cuspal Flexure, Depth-of-cure, and Mechanical Properties. Oper Dent 2019;44:E97-E104.

15. Faria-E-Silva AL, Pfeifer CS. Delayed photo-activation and addition of thio-urethane: Impact on polymerization kinetics and stress of dual-cured resin cements. J Dent 2017;65:101-109.

16. Boaro LC, Brandt WC, Meira JB, Rodrigues FP, Palin WM, Braga RR. Experimental and FE displacement and polymerization stress of bonded restorations as a function of the C-Factor, volume and substrate stiffness. J Dent 2014;42:140-148.

17. Feng L, Suh BI. A mechanism on why slower polymerization of a dental composite produces lower contraction stress. J Biomed Mater Res B Appl Biomater 2006;78:63-69.

18. Alqudaihi FS, Cook NB, Diefenderfer KE, Bottino MC, Platt JA. Comparison of Internal Adaptation of Bulk-fill and Increment-fill Resin Composite Materials. Oper Dent 2019;44:E32-E44.

19. Bandéca MC, El-Mowafy O, Saade EG, Rastelli ANS, Bagnato VS, Porto-Neto ST. Changes on degree of conversion of dual-cure luting light-cured with blue. LED Laser Physics 2009;19:1050-1055.

20. Schoerpf $S$, Catel $Y$, Norbert $M$, Gorsche $C$, Liska R. Enhanced reduction of polymerizationinduced shrinkage stress: Via combination of radical ring opening and addition fragmentation chain transfer. Polymer Chemistry 2019;10:1357-1366.

21. Soares CJ, Faria-E-Silva AL, Rodrigues MP, Vilela ABF, Pfeifer CS, Tantbirojn D, Versluis A. Polymerization shrinkage stress of composite resins and resin cements - What do we need to know? Braz Oral Res 2017;28:e62.

22. Bunek. Simplifying composite restorations. Dental Advisor 2017:2-11, www.dentaladvisor.com/pdf-download/?pdf_url=wp-content/uploads/2017/03/NOL-34-02restorations-WHOLE-ISSUE-Web.pdf . Accessed 26 March 2020.

23. Wang R, Wang Y. Depth-dependence of Degree of Conversion and Microhardness for Dualcure and Light-cure Composites. Oper Dent 2020;45:396-406. 
24. Nayyer M, Zahid $S$, Hassan SH, Mian SA, Mehmood S, Khan HA, Kaleem M, Zafar MS, Khan AS. Comparative abrasive wear resistance and surface analysis of dental resin-based materials. Eur J Dent 2018;12:57-66.

25. Tomaszewska IM, Kearns JO, Ilie N, Fleming GJ. Bulk fill restoratives: to cap or not to cap--that is the question? J Dent 2015;43:309-316.

Received: $15 / 05 / 2021$

Accepted: 13/09/2021 